\title{
The Correlation of Blood Parameters with Size in Cases of Neoplastic Tumor
}

\author{
Edyta Wolny-Rokicka ${ }^{1,2 *}$, Jerzy Wydmański ${ }^{3}$, Andrzej Tukiendorf ${ }^{4}$, Piotr Mróz ${ }^{5}$, \\ Katarzyna Gramacka ${ }^{3}$, Agnieszka Namyst-Kaletka ${ }^{3}$, Jakub Lipiński ${ }^{6}$, Agnieszka \\ Zembroń-Lacny²
}

\begin{abstract}
Purpose: This study aimed to evaluate the impact of tumor volume on platelet counts (PLT) and mean platelet volume (MPV) and involve these parameters on overall survival. Methods: It is a retrospective study of 99 patients with lung cancer (confirmed histologically or cytologically). Sixty-six patients underwent radical operating treatment and 33 patients had only biopsies - due to the inoperable status of tumor According to the histopathology profile: non-small cell carcinoma $-23 \%$, adenocarcinoma - $23 \%$, squamous - $36 \%$, small cell carcinoma $-11 \%$, carcinoid $-6 \%$. The overall survival was measured from the time of surgery to last observation or death. The tumor's size was established based on information from histopathology protocol by using model for the ellipsoid ( $V=4 / 3 \pi \mathrm{r}$ abc). Results: $\mathrm{KM}$ median survival time after surgery was 20 months $(95 \%$ C.I. $=16-42)$. The survival time depends significantly on: Tumor feature, MPV ( $\mathrm{p}=0.03, \mathrm{p}=0.04)$. Patients with normal PLT levels have longer survival time (median: 11 months) than thrombocytosis group (9.5) ( $\mathrm{p}=0.6$ ). Following both the PLT and MPV, a change-point that is equal to approximately $18.5 \mathrm{~cm}^{3}$ (approx. $3.3 \mathrm{~cm}$ in diameter) stands for a segmented relationship between tumor volume and analyzed blood indicators. Conclusions: After an overstepping of the change-point of tumor volume inflammatory processes start and they are associated with poor prognosis. MPV may be a valuable biomarker for the diagnosis and follow up of various types of carcinoma.
\end{abstract}

Keywords: Change-point analysis- lung cancer- mean platelet volume- prognostic factors- tumor volume

Asian Pac J Cancer Prev, 20 (1), 53-57

\section{Introduction}

Platelets are elements in the blood which play the main role in the coagulation cascade. The association between elevated platelet count (PLT) and malignancies was recognized over a century ago (Riess, 1872). The pathogenesis of thrombocytosis in malignancy has not yet been clarified. Although it can be symptomless (particularly when it is a secondary reaction), it can predispose patients to thrombosis. In patients with cancer, the prevalence of thrombocytosis ranges from $10 \%$ to 57\% (Sierko and Wojtukiewicz, 2004). As recognized by Kabir and Darr in 1995 and Wu et al in 1996 (Kabir and Darr, 1995; Wu et al., 1996) tumor cells secrete humoral factors may eventually lead to thrombocytosis (WolnyRokicka et al., 2018). The correlation between parameters such as PLT, mean platelet volume (MPV) and different cancer types has been previously investigated, but the correlation between PLT and MPV and tumor size has not yet been studied. The present study aims to find the relation between the tumor size PLT and MPV in lung cancer cases.

\section{Materials and Methods}

\section{Patient's characteristics}

This study was approved by the Ethics Committee at the Medical Council in The Regional Medical Chamber, Zielona Góra, Poland. It is a retrospective study of 99 patients with lung cancer (confirmed histologically or cytologically) who were inpatients of the Clinic of Thoracic Surgery at the Regional Clinical Hospital in Zielona Gora between 2009 and 2010. The subjects participating in the study provided both oral and written

${ }^{1}$ Radiotherapy Development Department in Provincial Multidisciplinary Hospital in Gorzów Wielkopolski, ul Dekerta 1, 66-400 Gorzow Wielkopolski LLC, ${ }^{2}$ University of Zielona Góra, Faculty of Medicine and Health Sciences, ul. Zyty 28, ${ }^{5}$ University of Zielona Góra, Faculty of Computer Science, Electrical Engineering and Automation, Institute of Metrology, Electronics and Computer Science, ul. Prof. Z. Szafrana, ${ }^{6}$ Laboratory, Regional Clinical Hospital in Zielona Góra ul, Zyty, Zielona Góra, ${ }^{3}$ Department of Radiotherapy, Center of Oncology-Maria Skłodowska-Curie Memorial Institute, Branch in Gliwice, ul Wybrzeża Armii Krajowej 15,44-101 Gliwice, ${ }^{4}$ Social Medicine Department, Medical University in Wroctaw, ul. Bujwida 44, 50-345 Wroctaw, Poland. *For Correspondence: edyta.wolny@gmail.com 
informed consent. The disease stage was defined based on clinical and physical examinations such as thoracic computed tomography (CT), brain CT or magnetic resonance imaging, abdominal ultrasonography, bone scintigraphy and/or positron emission tomography-CT. The histopathological data were determined according to the Union for International Cancer Control (UICC) TNM classification (Sobin et al., 2010). The tumor's size was established based on information from histopathology protocol by using model for the ellipsoid ( $\mathrm{V}=4 / 3 \pi \mathrm{r}$ $a b c)$. The exclusion criteria were as follows: (i) a history of secondary tumors(s); (ii) an active infection; (iii) disease with previous chemo/radiotherapy history; (iv) the World Health Organization performance status of 4 (i.e. completely disabled, unable to undertake any self-care, totally confined to bed or chair). All subjects were patients with a primary lung cancer before any chemotherapy or radiotherapy. They were included in the study consecutively as they reported to the thoracic surgery department. 66 patients underwent radical operating treatment and 33 patients had only biopsies - due to the inoperable status of the tumor. The group consisted of $81 \%$ male and $19 \%$ female subjects. According to the histopathology profile: non-small cell carcinoma $-23 \%$, adenocarcinoma $-23 \%$, squamous $-36 \%$, small cell carcinoma $-11 \%$, carcinoid $-6 \%$. The overall survival was measured from the time of surgery to last observation or death (the information about the date of death was obtained from the National Health System). The last follow-up date was July 31, 2016.

Biochemical assays: The venous blood samples were drawn from peripheral blood before the medical procedure (surgery) and evaluated by measuring the complete blood count (CBC) with a hematology analyzer (Abbott CD3700, CD RUBY, USA). The reference value in our hospital for this parameters is: PLT: $140-420$ tys/ $\mu \mathrm{l}$, MPV:

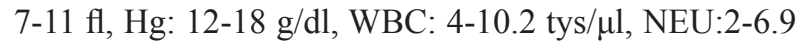

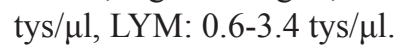

\section{Results}

\section{Statistical analysis}

Demographic features and parameters such as PLT, MPV, tumor volume (TV) results are presented in Table 1. The overall and median survival time was analyzed using Kaplan Meier (KM) methodology. KM median survival time after surgery was 20 months $(95 \%$ C.I. $=16-42)$, (Figure 1). The survival time depends significantly on: Tumor feature, MPV $(\mathrm{p}=0.03, \mathrm{p}=0.04)$. Patients with normal PLT levels have longer survival time (median: 11 months) than thrombocytosis group (9.5) $(\mathrm{p}=0.6)$. In order to predict the future risk of death, an impact of the tumor
Table 1. Demographic Features and Mean Platelet Volume (MPV), Platelet (PLT), Tumor Volume (TV) Results

\begin{tabular}{lccc}
\hline Factor & mean \pm S.D. & Median & Range \\
\hline age & $62.9 \pm 8.1$ & 63 & $41-81$ \\
V [cm3] & $48.9 \pm 107.7$ & 15.8 & $0.5-825$ \\
PLT [G/L] & $350 \pm 130$ & 324 & $150-734$ \\
MPV [fl] & $8.1 \pm 1.9$ & 7.4 & $5.1-13.2$ \\
\hline
\end{tabular}

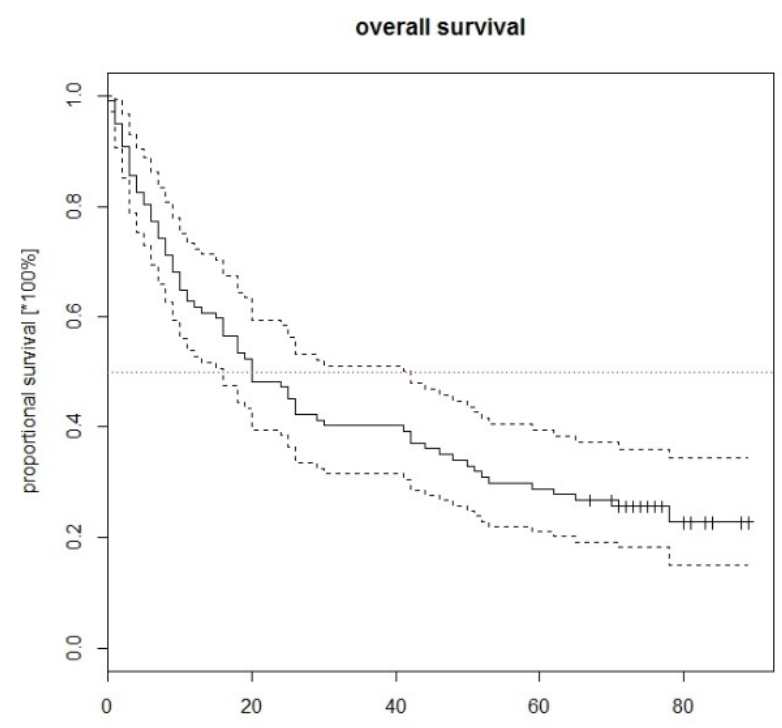

Figure 1. Kaplan Meier (KM) Median Survival Time after Surgery

volume on the overall survival in patients was estimated using time-dependent receiver operating characteristic (ROC) analysis (Blanche et al., 2013), (Table 2 and Figure 2). A linear approximation of the estimated parameters (Table 2) gives p-value $=0.0002$ for the intercept and 0.2672 for the slope; therefore, the regression coefficients stand for a stable trend of the area under curves (AUC) over the time of observation of patients. The volumetric characteristic of the overall survival in patients was plotted using 'classical' (time independent) ROC analysis (Figure 3), (Robin et al., 2011). Tumor volume ROC curve analyses were performed to identify the optimal

Table 2. Time Dependent AUC

\begin{tabular}{lc}
\hline Time [month] & AUC (S.E.) [\%] \\
\hline 9 & $76.02(6.23)$ \\
18.9 & $65.01(6.82)$ \\
35.5 & $68.73(6.59)$ \\
63.7 & $66.86(7.13)$ \\
74.6 & $65.08(8.07)$ \\
\hline
\end{tabular}

Table 3. Segmented Regression Parameters: Platelet and Mean Platelet Volume (PLT and MPV)

\begin{tabular}{lccccc}
\hline Indicator & Change Point (S.E.) & Slope & Estimate & $95 \%$ C.I. & p-value \\
\hline PLT & $17.8(5.9)$ & I & $7.81(3.45)$ & $(0.91,14.71)$ & $<0.05$ \\
& & II & $-0.23(0.14)$ & $(-0.51,0.06)$ & N.S. \\
MPV & $19.1(9.8)$ & I & $-0.07(0.05)$ & $(-0.18,0.03)$ & N.S. \\
& & II & $0.0052(0.0024)$ & $(0.0004,0.01)$ & $<0.05$ \\
\hline
\end{tabular}




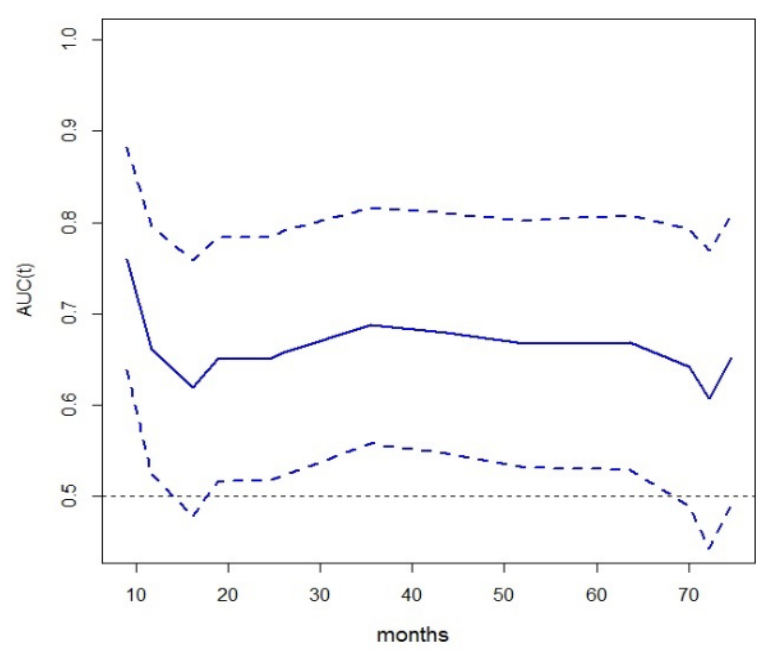

Figure 2. Time Dependent AUC (with 95\% C.I.)

Table 4. Cox Regression was Used to Analyze Tumor Volume (TV) as a Independent Prognostic Risk Factors. The better fit regression (a large z statistic) was established

\begin{tabular}{lccc}
\hline $\begin{array}{l}\text { change-point } \\
\text { volume }\left[\mathrm{cm}^{3}\right]\end{array}$ & HR $(95 \% \mathrm{CI})$ & z statistic & p-value \\
\hline 2.7 & $5.73(1.34,24.4)$ & 2.41 & 0.016 \\
18.5 & $2.21(1.21,4.02)$ & 2.63 & 0.008 \\
\hline
\end{tabular}

cut-off values of the TV level. It can be seen in Figure 3 , that the optimal cut-off of tumor volume to predict death in patients was established of around $2.7 \mathrm{~cm} 3$ (approx. $1.7 \mathrm{~cm}$ in diameter). However, from clinical and diagnostic points of view, the estimated size seems to be of minor usefulness (specificity $=35 \%$ is relatively low). To estimate the change-point, a segmented (brokenline) relationship platelet and mean platelet volume (PLT and MPV) was applied in the statistical analysis (Muggeo, 2003). Following both the PLT and MPV, a change-point that is equal to approximately $18.5 \mathrm{~cm}^{3}$ (approx. $3.3 \mathrm{~cm}$ in diameter) stands for a segmented

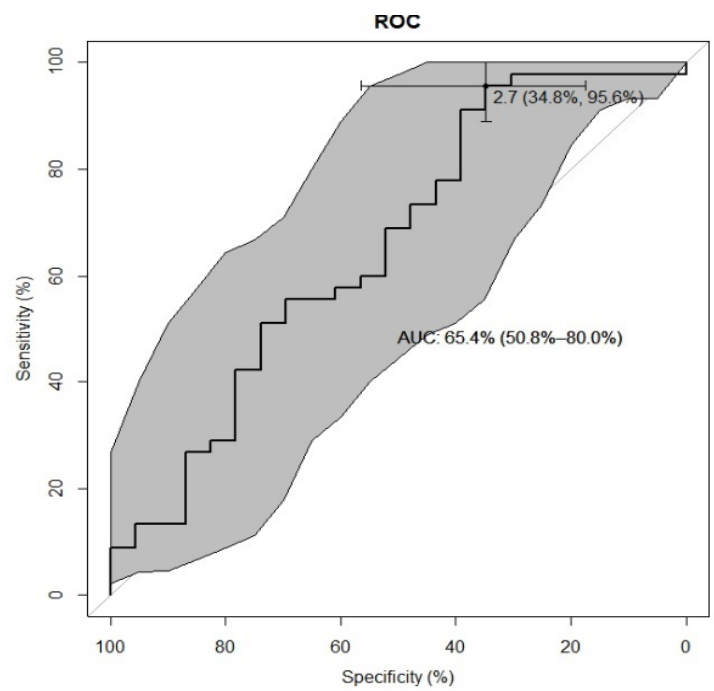

Figure 3. Time Independent AUC (with 95\% C.I.).TV ROC (receiver operating charakteristic) curve analysis to a identyfity optimal cut-off values of the TV level.

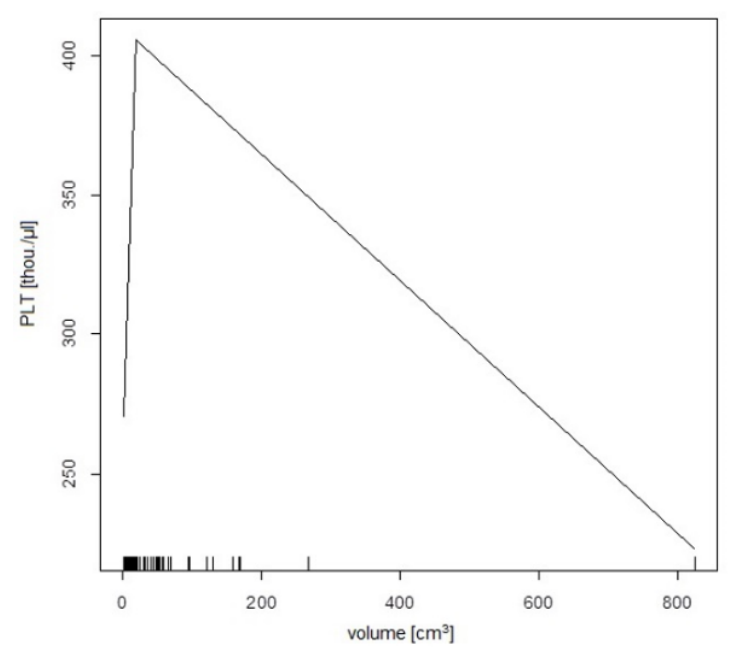

Figure 4. PLT vs. Tumor Volume (Segmented Regression)

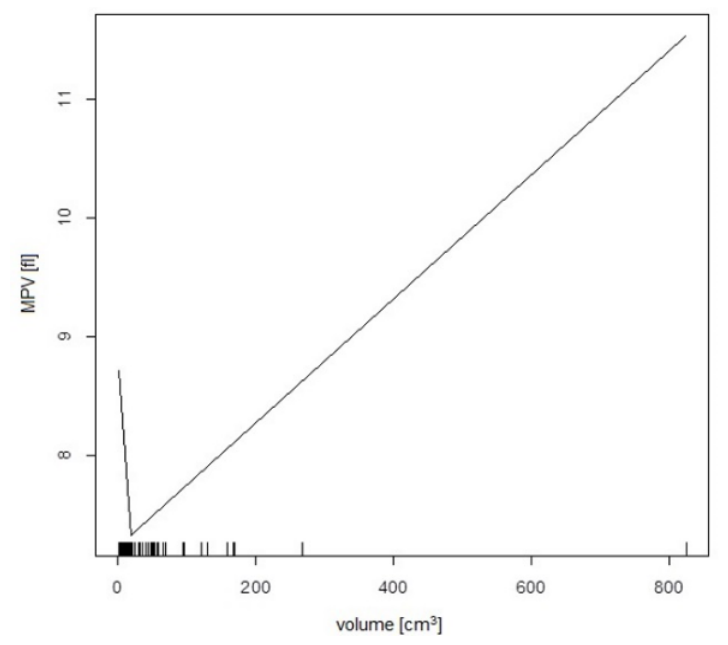

Figure 5. MPV vs. Tumor Volume (Segmented Regression)

relationship between tumor volume and analyzed blood indicators. The effects of tumor volume on PLT and MPV are presented graphically in plots in Figure 4 and Figure 5, respectively. Finally, for the volume change-points, the Cox regression was used to establish model fitting (Table 4). Following the reported $\mathrm{z}$ statistic and $\mathrm{p}$-value (see Table 4 estimates), a better regression fit was established for the $18.5 \mathrm{~cm} 3$ volume $(3.3 \mathrm{~cm}$ diameter) of the tumor in predicting the overall survival in patients. The outcome can be put to practical use in clinical measures. The computation was performed in the $\mathrm{R}$ statistical platform (R Core Team R, 2018).

\section{Discussion}

Over the past decades, tumor characteristic quantified through volumetric analysis has proven useful for establishing prognosis in the overall survival of patients. The present study investigated whether PLT and MPV at diagnosis or before surgery procedures have a predictive value (ie. tumor volume in predicting survival in lung cancer patients). Four types of analysis were used and the same point was found - the tumor volume $18.5 \mathrm{~cm}^{3}$ - after which the parameters of MPV and PLT are dichotomous. 
MPV was increasing and PLT was decreasing. MPV is the platelet activity marker that is most commonly used to evaluate inflammatory processes and malignancies. Platelets with larger MPV have granules containing more mediators and thus play a bigger role in the progress of cancer development (Kisucka et al., 2006; Mantovani et al., 2008). Authors Eryilmaz et al., (2015) and Li et al., (2014) in their studies demonstrated that cancer patients have higher MPV levels compared to the controls (head and neck and colon cancer patients studies, respectively). In another study, Wolny-Rokicka et al., (2018) found the decrease of MPV after radiotherapy treatment. Li et al., (2014) showed that there is no difference between the groups with metastasis and without metastasis in terms of MPV. But, MPV was lower in I-II stages compared to III-IV stages of cancer the same as in Oncel et al., (2016) study with patients in early-stage lung cancer. Baldane et al., (2015) in their study of papillary thyroid carcinoma patients presented a significant drop in MPV levels after surgery. The same as when applied an anti-angiogenic agent bevacizumab which reduced the MPV levels in metastatic colon cancer patients (2012). In another study with lung cancer patients, it was found that MPV is not changed depending on tumor stage and histologic types (Kemal et al., 2014). Tumor cells release procoagulant, fibrinolytic factors, mediators, proteases, cytokines which have activated platelets (Noble and Pasi, 2010; Bagoly, 2015). Platelets initiate the development of the inflammatory process. Inflammation is responsible for the increased MPV. In the present study opposite trend of the PLT and MPV broken-lines was obtained. With the increase of the tumor volume after change-point $\left(18,5 \mathrm{~cm}^{3}\right)$ the MPV increases similarly to the above studies and PLT slowly decreases with the increase of the tumor volume. It is a finding contrary to the Pedersen et al., (1996) and Dvorak et al., (1994) study. There are also some other studies suggesting that there is no change in PLT (Oncel et al., 2016). In normal circumstances, there is an opposite relationship between the number and volume of PLT in order to support a constant mass of circulating platelets. When PLTs are decreased, megakaryocytes in the bone marrow are stimulated by thrombopoietin. And their nucleuses are transformed into hyper lobes which have a high DNA content. PLTs play an important and multifaceted role in cancer as described by the following authors: Inagaki et al., (2014), Li et al., (2014), Ceylan et al., (2015). First, since larger platelets are more reactive than the smaller ones and more responsive against endogenous and exogenous stimuli, they may cause consumption of these cells (Inagaki et al., 2014; Li et al., 2014). The other reason might be that small platelet may be more prominent in circulation, depending on the destruction and sequestration of platelets in the active inflammation (Ceylan et al., 2015). Moreover, activated platelets play a pivotal role in cancer metastasis through the release of cytokines, chemokines, and expression of several adhesion receptors (Borsig, 2008). The tumor can "consume" these factors causing changes in both thrombosis and bleeding. These phenomena were described by Tafur et al., (2012) who compared the processes of thrombosis and bleeding as higher in patients with active cancer. The change-point of tumor volume $\left(18.5 \mathrm{~cm}^{3}\right)$ is correlated with TNM classification. It is described as an early stage of disease e.g. T1, T2. In conclusion, the result (approx. $3.3 \mathrm{~cm}$ in diameter) stands for a segmented relationship between the tumor volume and analyzed blood indicators and is not a strong statistical evidence but seems to be very practical clinically. After an overstepping of the change-point of tumor volume, inflammatory processes start and they are associated with poor prognosis.

\section{References}

Bagoly Z (2015). Cancer and thrombosis: a fresh look at an old story. Thromb Res, 136, 1-2.

Baldane S, Ipekci SH, Sozen M, Kebapcilar L (2015). Mean platelet volume could be a possible biomarker for papillary thyroid carcinomas. Asian Pac J Cancer Prev, 16, 2671-4.

Blanche P, Dartigues J, Jacqmin-Gadda H (2013). Estimating and comparing time dependent areas under receiver operating characteristic curves for censored event times with competing risks. Stat Med, 32, 5381-9.

Borsig L (2008). The role of platelet activation in tumor metastasis. Expert Rev Anticancer Ther, 8, 1247-55.

Ceylan B, Aslan T, Cinar A, Ruhkar Kurt A, Akkoyunlu Y (2015). Can platelet indices be used as predictors of complication in subjects with appendicitis?. Wien Klin Wochenschr, 15, 760-4.

Dvorak HF (1994). Abnormalities of hemostasis in malignancy. In: Colman RW, Hirsch J, Marder VJ et al, eds. Hemostasis and Thrombosis. Basic Principles and Clinical Practice, Third edition. Philadelphia: JB Lippincott, pp 1238-54.

Eryilmaz A, Basal Y, Omurlu IK (2015). Can head and neck cancers be detected with mean platelet volume?. Asian Pac J Cancer Prev, 16, 7045-7.

Inagaki N, Kibata K, Tamaki T, Shimizu T, Nomura S (2014). Prognostic impact of the mean platelet volume/platelet count ratio in terms of survival in advanced non-small cell lung cancer. Lung Cancer, 83, 97-101.

Kabir S, Darr GA (1995). Serum levels of interleukine-1, interleukine- 6 and tumor necrosis factor-alpha in patients with gastric carcinoma. Cancer Lett, 95, 207-12.

Kemal Y, Yucel I, Ekiz K, et al (2014). Elevated serum neutrophil to lymphocyte and platelet to lymphocyte ratios could be useful in lung cancer diagnosis. Asian Pac J Cancer Prev, $15,2651-4$.

Kisucka J, Butterfield CE, Duda DG, et al (2006). Platelets and platelet adhesion support angiogenesis while preventing excessive hemorrhage. Proc Natl Acad Sci U S A, 103, 855-60.

Li J-Y, Li Y, Jiang Z, Wang R-T, Wang X-S (2014). Elevated Mean Platelet Volume is associated with colon cancer. Asian Pac J Cancer Prev, 15, 10501-4.

Mantovani A, Allavena P, Sica A, Balkwill F (2008). Cancer-related inflammation. Nature, 454, 436-44.

Muggeo V (2003). Estimating regression models with unknown change-points. Stat Med, 22, 3055-71.

Mutlu H, Berk V, Karaca H, et al (2012). Treatment regimen with bevacizumab decreases mean platelet volume in patients with metastatic colon cancer. Clin Appl Thromb Hemost, 18, 546-8.

Noble S, Pasi J (2010). Epidemiology and pathophysiology of cancer-associated thrombosis. Br J Cancer, 102, 2-9.

Oncel M, Kiyici A, Oncel M, et al (2016). Evaluation of platelet indices in lung cancer patients. Asian Pac J Cancer Prev, 16, 7599-602. 
Pedersen LM, Milman N (1996). Prognostic significance of thrombocytosis in patients with primary lung cancer. Eur Respir J, 9, 1826-30.

R Core Team R (2016). A language and environment for statistical computing. R Version 3.5.0. Vienna: R Foundation for Statistical Computing, http://cran.r-project.org.

Riess L (1872). Zur pathologischen anatomie des blutes. Anat Physiol Wissensch Med, 39, 237-49.

Robin X, Turck N, Hainard A, et al (2011). pROC: an open-source package for $\mathrm{R}$ and $\mathrm{S}+$ to analyze and compare ROC curves. BMC Bioinformatics, 12, 77.

Sierko E, Wojtukiewicz MZ (2004). Platelets and angiogenesis in malignancy. Semin Thromb Hemost, 30, 95-108.

Sobin LH, Gospodarowicz MK, Wittekind C (2010). International Union Against Cancer (UICC): TNM classification of malignant tumors. 7th edition. John Willkey and Sons, Ltd., UK.

Tafur AJ, Wysokinski WE, McBane RD, et al (2012). Cancer effect on periprocedural thromboembolism and bleeding in anticoagutaled patients. Ann Oncol, 23, 1998-2005.

Wolny-Rokicka E, Brzezniakiewicz-Janus K, Wydmański J, Tukiendorf A, Zembroń-Łacny A (2018). Analysis of haemostasis biomarkers in patients with advanced stage lung cancer during hypofractionated radiotherapy treatment. J Int Med Res, 46, 1876-83.

Wolny-Rokicka E, Tukiendorf A, Wydmański J, Zembroń-Łacny A (2018). The potential of the quick detection of selectins using Raman Spectroscopy to discriminate lung cancer patients from healthy subjects. $J$ Spectrosc, 9, 1-5.

Wolny-Rokicka E, Wydmański J, Tukiendorf A, Mróz P, Zembroń-Łacny A (2018). Study of appraisal of basic haemostatic markers in lung cancer patients in follow up after radiotherapy. Med Sci Monit, 24, DOI: 10.12659/ MSM.91028.

Wu CW, Wang SR, Chao MF, et al (1996). Serum interleukine-6 levels reflect disease status of gastric cancer. $\mathrm{Am} \mathrm{J}$ Gastroenterol, 91, 1417-22.

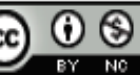

This work is licensed under a Creative Commons AttributionNon Commercial 4.0 International License. 